\title{
ANÁLISIS TERMOFÍSICO DE MORTEROS CEMENTO ARENA 1:4 PARA CONSTRUCCIÓN, SUSTITUYENDO ARENA POR PERLITA SINTÉTICA
}

\author{
Thermophysical analysis of mortars 1: 4 cement-sand for \\ construction, replacing sand for synthetic perlite.
}

\section{EPISTEMUS}

ISSN: 2007-8196 (electrónico)

ISSN: 2007-4530 (impresa)

\section{Ana Cecilia Borbón Almada ${ }^{1}$ \\ Dagoberto Burgos Flores 2 \\ Samuel Castro Brockman 3 \\ Arturo Duarte Gaxiola 4 \\ Jesús Quintana Pacheco 5}

Recibido: 15 de marzo de 2017

Aceptado: 26 de mayo de 2018

Autor de Correspondencia:

Dra. Ana Cecilia Borbón Almada

Correo: acborbon@dicym.uson.mx

\section{Resumen}

Las mezclas para morteros convencionales utilizados en la industria de la construcción, suelen ser a base de cemento, agua y agregados, principalmente arena. Actualmente se observa una tendencia en la utilización de morteros más ligeros, menos densos y menos conductivos para efectos de aplicaciones con más ventajas en su manejo y capacidad aislante. En este proyecto se experimenta con la sustitución de arena en diferentes porcentajes, por materiales aligerantes, con la intención de probar el comportamiento termofísico en función de las variaciones en el peso volumétrico, densidad, esfuerzo a la compresión y conductividad térmica. Se han encontrado las proporciones que cumplen con la condición de ligereza y esfuerzo a la compresión, que los califica como materiales utilizables en aplicaciones específicas en la industria de la construcción sustentable, que se demanda hoy en día en México.

Palabras clave: Morteros,Conductividad térmica, Esfuerzo a la compresión.

\section{Abstract}

The conventional mortar mixtures used in the construction industry are usually based on cement, water and aggregates, mainly sand. There is a trend in the use of lighter, less dense and less conductive mortars for applications with more advantages in their handling and insulation capacity. In this project, it is experimented with the substitution of sand in different percentages, by lightening materials, with the intention to test the thermophysical behavior in function of the variations in the volumetric weight, density, compressive stress and thermal conductivity. They have found the proportions that meet the condition of lightness and effort to compression, which makes them usable materials in specific applications in the construction industry that is demanded today in Mexico.

Keywords: Mortars, Thermal conductivity, Compressive stress. 


\section{INTRODUCCIÓN}

En la industria de la construcción en México, se utilizan materiales para conformar mezclas ya sea para la elaboración de concretos, lechadas o morteros, los cuales se forman básicamente de agua, cemento y agregados en diferentes proporciones dependiendo de su aplicación.

Estas mezclas tienen diferentes usos; desde los concretos para elementos estructurales, los morteros en enjarres, enlucidos o rellenos y las lechadas para detalles de construcción.

Este trabajo presenta los resultados de las variaciones de las principales características físicas del mortero cemento arena 1:4, utilizado para enjarres, pegados y elaboración de tabiques, sustituyendo arena por perlita sintética en las proporciones de $20 \%, 40 \%, 60 \%, 80 \%$ y $100 \%$. Entre los resultados más relevantes se destacan los valores del esfuerzo a la compresión para las diferentes proporciones, así como la conductividad térmica.

Los materiales constructivos juegan un papel importante en la respuesta térmica que un edificio puede dar; sin embargo, la parte estructural de los mismos debe revisarse y conjugar una combinación de aquellos materiales que sean capaces de soportar esfuerzos mecánicos y que también ofrezcan posibilidades de ser más ligeros, menos densos y menos conductivos.

Para atender la normatividad mexicana, con respecto a las características de los materiales, se cuenta con la norma mexicana NMX-C-486-ONNCCE [1], que determina el esfuerzo a la compresión que debe soportar un mortero o tabique de mampostería de acuerdo al tipo de aplicación.

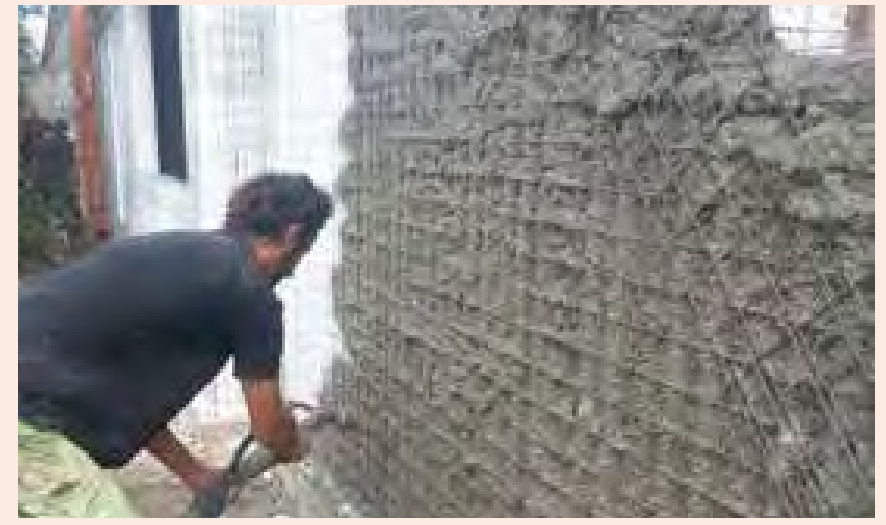

Desde el punto de vista de la capacidad aislante del material, también existe una norma mexicana NMX-C460-ONNCCE-2009 [2], que revisa la resistencia térmica de elementos constructivos, basado principalmente en los valores de la conductividad térmica.

En este artículo se muestran los resultados experimentales de un proyecto de investigación, donde se han diseñado y fabricado, morteros aligerados con perlita sintética de tipo comercial.

A partir de los diseños propuestos para las mezclas aligeradas con diferentes porcentajes de sustitución, se fabrican los morteros verificando el cumplimiento de las normas para su comportamiento en estado fresco (fluidez y contenido de aire) así como en estado seco para probar los esfuerzos a la compresión y conductividad térmica, lo que permite establecer relaciones para determinar su respuesta con respecto a estos parámetros y posibles aplicaciones en la práctica constructiva.

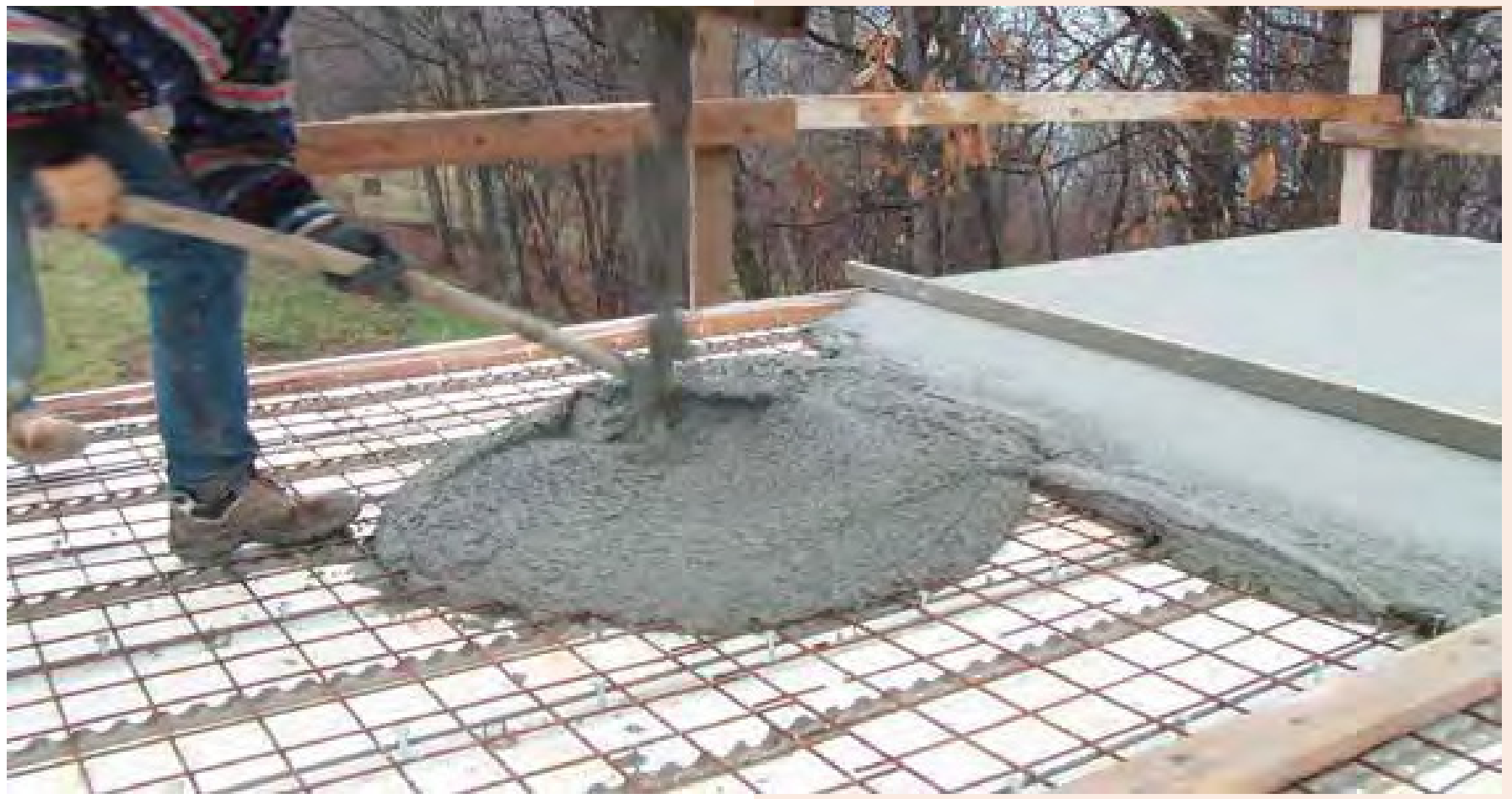

Ana Cecilia Borbón Almada et al.: Análisis termofísico de morteros cemento arena... 


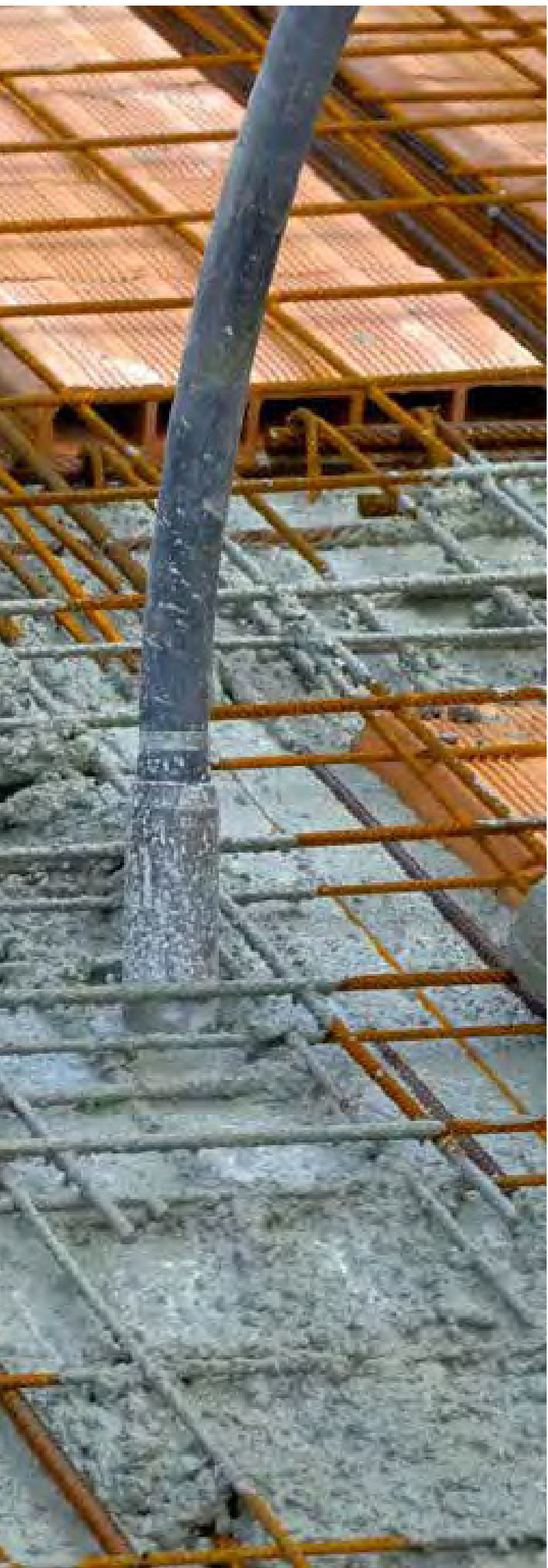

\section{ANTECEDENTES}

Las técnicas para sustituir algunos materiales convencionales de construcción, particularmente los agregados como la arena, por otros menos densos que proporcionen propiedades que mejoren la ligereza en cuanto a peso y densidad volumétrica, son comunes en la literatura; sin embargo, no lo son tanto aquellos estudios que se refieran a analizar y sobre todo evaluar experimentalmente la conductividad térmica, que es un parámetro importante cuando se trata de descifrar la capacidad de aislamiento que puedan proporcionar estos materiales.

Existen algunas investigaciones donde se muestra el resultado de propiedades de mezclas aligeradas con materiales diversos o de tipo alternativo, como es el caso de Barros et al. [3] que estudió la utilización de fibra de vidrio para concreto reforzado. También se han estudiado sustituciones de materiales como el caucho, por parte de Bustamante e. al [4]. Del Río et al. [5] trabajó con arcillas expandidas y Marcos J. et al. [6] con materiales alternativos, basando sus resultados en propiedades mecánicas de los morteros.

El desarrollo de este proyecto se basa en la utilización de materiales industrializados y vigentes en el mercado de la construcción, dada la falta de información que existe del comportamiento termo físico de los mismos en forma de mezclas líquidas y endurecidas modificadas, en función de la utilización de materiales aligerantes de peso y densidad, así como de una baja conductividad térmica. Al no existir esta información de manera generalizada y probada en el mercado de la construcción, este proyecto pretende mostrar estas características con materiales aligerantes ya industrializados y comerciales donde existe una gran demanda por el conocimiento de estas características y sus aplicaciones.

\section{MÉTODO}

La matriz de experimentación que a continuación se presenta, contiene las cantidades ajustadas de agua, cemento, arena y el material que se utiliza para la sustitución en porcentajes desde $0 \%, 20 \%, 40,60 \%$, 80 , y $100 \%$. La tabla muestra las cantidades de materiales, como los son, el cemento CPC- 30- $\mathrm{R}$, arena común y perlita sintética, así como las cantidades de agua ajustadas, en un proceso de laboratorio, de acuerdo a lo expresado por Durán C. [7] que se basa en la teoría de Sánchez de Guzmán [8].

Tabla1. Tabla de diseño de mezclas para morteros aligerados con perlita sintética

\begin{tabular}{|l|l|l|l|l|l|l|}
\hline Material & $\begin{array}{c}\text { Mezcla de } \\
\text { control }\end{array}$ & \multicolumn{5}{|c|}{$\begin{array}{c}\text { Sustitución de arena } \\
\text { por perlita sintética (\%) }\end{array}$} \\
\hline (KG) & & 20 & 40 & 60 & 80 & 100 \\
\hline Cemento & 6.218 & 6.218 & 6.218 & 6.218 & 6.218 & 6.218 \\
\hline Arena & 21.223 & 16.978 & 12.734 & 8.489 & 4.245 & 0.000 \\
\hline Perlita & 0.000 & 0.023 & 0.046 & 0.070 & 0.093 & 0.116 \\
\hline Agua & 4.578 & 3.900 & 3.510 & 3.360 & 3.100 & 3.054 \\
\hline
\end{tabular}

A partir del diseño, se fabrican las mezclas para un volumen de 15 litros, con las cantidades de materiales que se indican en la tabla 1, para las 6 variaciones de mortero aligerado con equipos convencionales de mezclado. 
Una vez elaboradas las mezclas, se realizan las pruebas de control en estado fresco, como lo son la prueba de fluidez y contenido de aire, verificando el cumplimiento de la normatividad para las mezclas en estado fresco (pruebas de control). Para las pruebas en estado endurecido se utilizan moldes de fraguado para probar los esfuerzos a la compresión y la conductividad térmica (pruebas para resultados), finalmente se comparan los resultados y se hacen recomendaciones para su uso.

\section{Preparación de los materiales y procedimiento}

Para la elaboración de las mezclas se utiliza arena con una densidad de $2.56 \mathrm{~g} / \mathrm{cm}^{3}$, absorción de $1.53 \%$, y módulo de finura de 2.80; cemento CPC $30 \mathrm{R}$ y agua potable, así como el material aligerante que consiste en una perlita sintética de tipo comercial con densidad de $0.014 \mathrm{~g} / \mathrm{cm}^{3}$. Se realizan las mezclas en cantidades de 15 litros, para cada tipo de mezcla o proporción. Se realizan las mezclas de forma convencional, en revolvedora de alimentación eléctrica de 70 litros, donde se requiere mezclar la perlita con el cemento completamente secos y agregar el agua de manera posterior para lograr un mezclado uniforme y evitar la segregación del material liviano, como se muestra en la figura 1.

Una vez elaboradas las mezclas, se realizan las pruebas en estado fresco de la medición de fluidez y contenido de aire, de acuerdo a la normatividad mexicana NMX-C-061[9] y NMX-C157 ONNCCE [10] en equipos especializados. Se verificó que todas las mezclas cumplan con una fluidez de $110 \%$ con una tolerancia de $\pm 5 \%$ y valores de contenido de aire menores al $20 \%$.

Se fabricaron moldes para vaciar los morteros tanto para las pruebas a la compresión que son moldes cúbicos de $5 \times 5 \times 5 \mathrm{~cm}$ y cilíndricos de $5 \mathrm{~cm}$ de diámetro. Se fabricaron también moldes cuadrados de $15 \times 15$ $\mathrm{cm}$ y de $4 \mathrm{~cm}$ de espesor para el vaciado de las mezclas con las que se midió la conductividad térmica.

Una vez elaboradas las muestras se dejan secar para probar a la compresión como una prensa a los 3,7 y 28 días de fraguado. Después de los 28 días, las muestras cuadradas se someten a un secado en horno a $100^{\circ} \mathrm{C}$ durante 24 horas y posteriormente son medidos para la conductividad térmica en un equipo especializado denominado Lamda meter EP 500 e [11], de acuerdo a la norma internacional ASTM-C-177 [12].
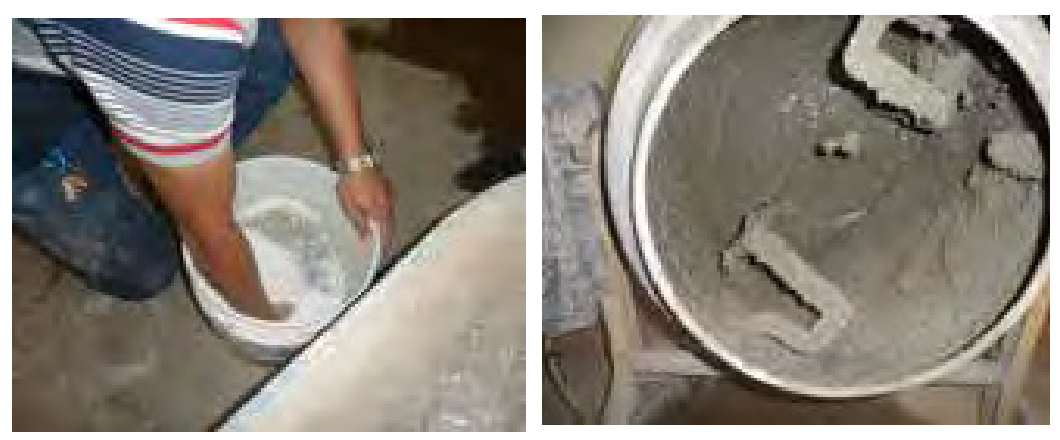

Figura 1. Mezcla de los materiales aligerados con perlita sintética 

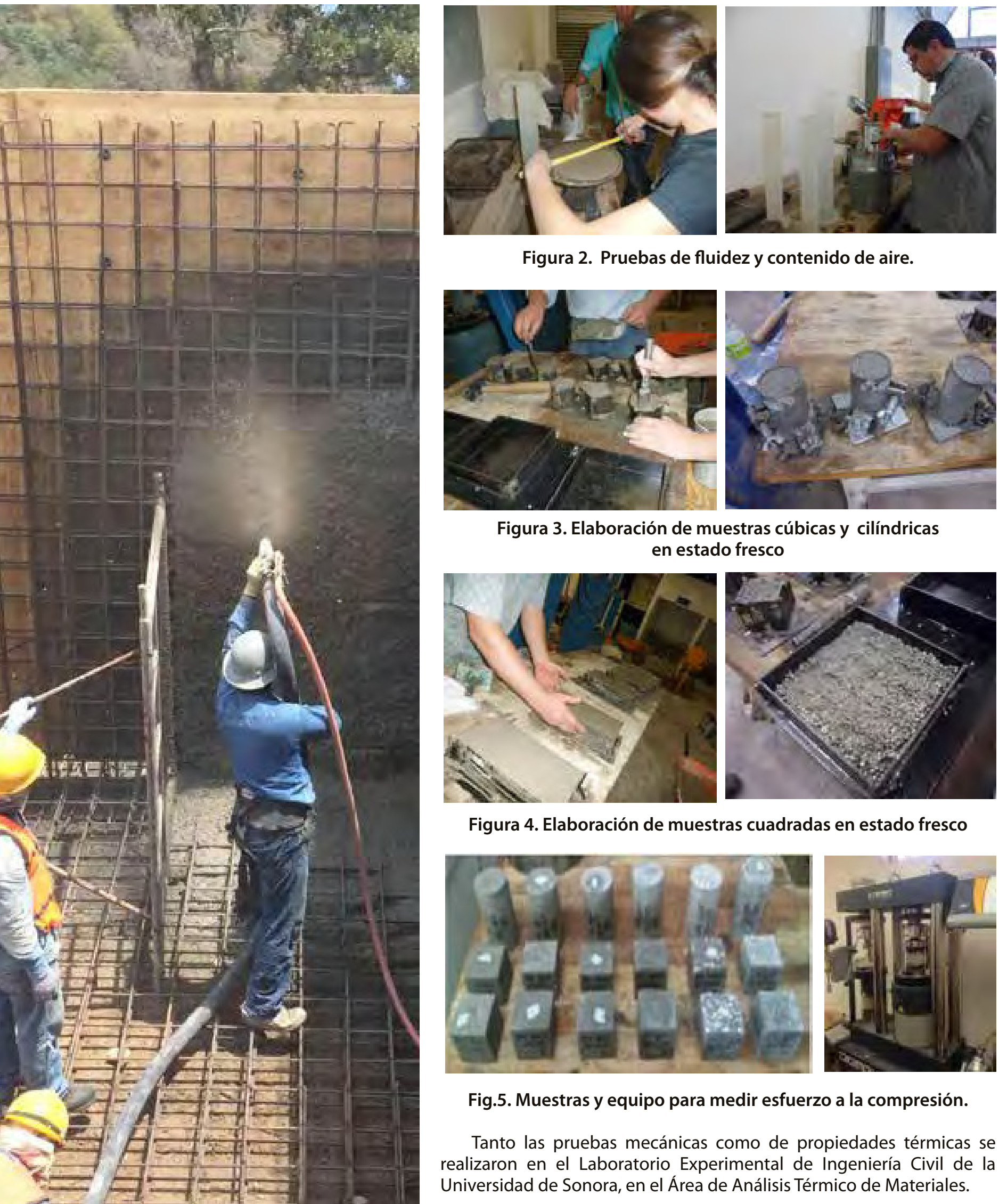

Figura 2. Pruebas de fluidez y contenido de aire.
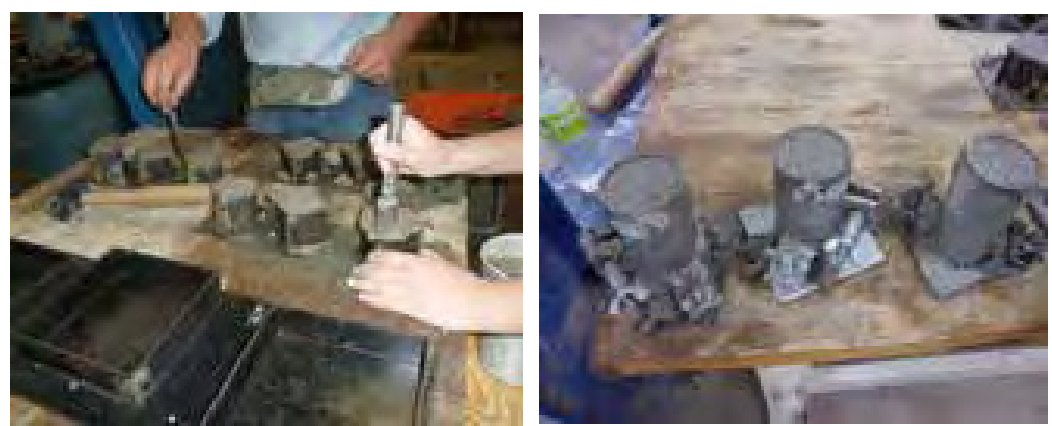

Figura 3. Elaboración de muestras cúbicas y cilíndricas en estado fresco

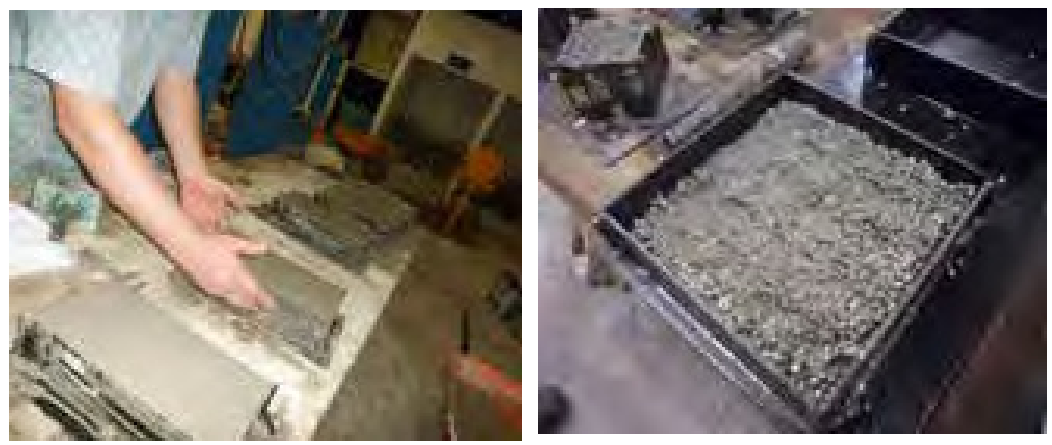

Figura 4. Elaboración de muestras cuadradas en estado fresco
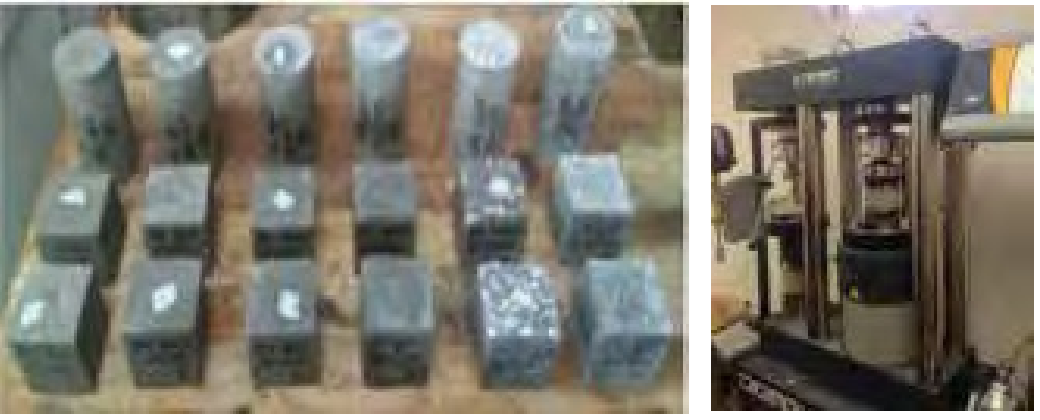

Fig.5. Muestras y equipo para medir esfuerzo a la compresión.

Tanto las pruebas mecánicas como de propiedades térmicas se realizaron en el Laboratorio Experimental de Ingeniería Civil de la Universidad de Sonora, en el Área de Análisis Térmico de Materiales. 

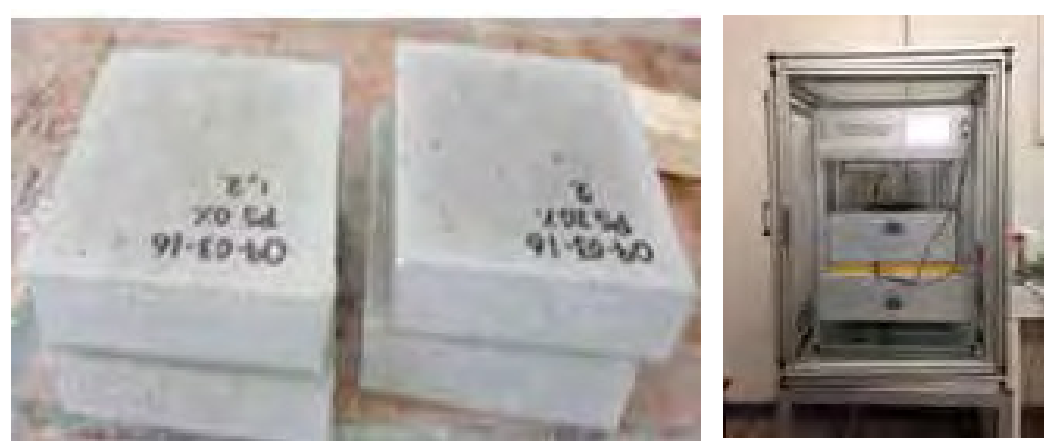

Fig.6. Muestras y equipo para medir conductividad térmica.

\section{RESULTADOS Y DISCUSIÓN}

En la figura 7, se pueden observar los resultados de los esfuerzos a la compresión, las edades y los diferentes porcentajes de sustitución de las mezclas aligeradas con pelita sintética. De acuerdo a estos resultados se observa que la resistencia a la compresión aumenta de acuerdo a la edad del fraguado; este comportamiento es característico del comportamiento de las mezclas. Para el caso de los resultados en relación al esfuerzo a la compresión y el porcentaje de sustitución, se observa una disminución en el esfuerzo a la compresión en la media que se aumenta el porcentaje de sustitución.

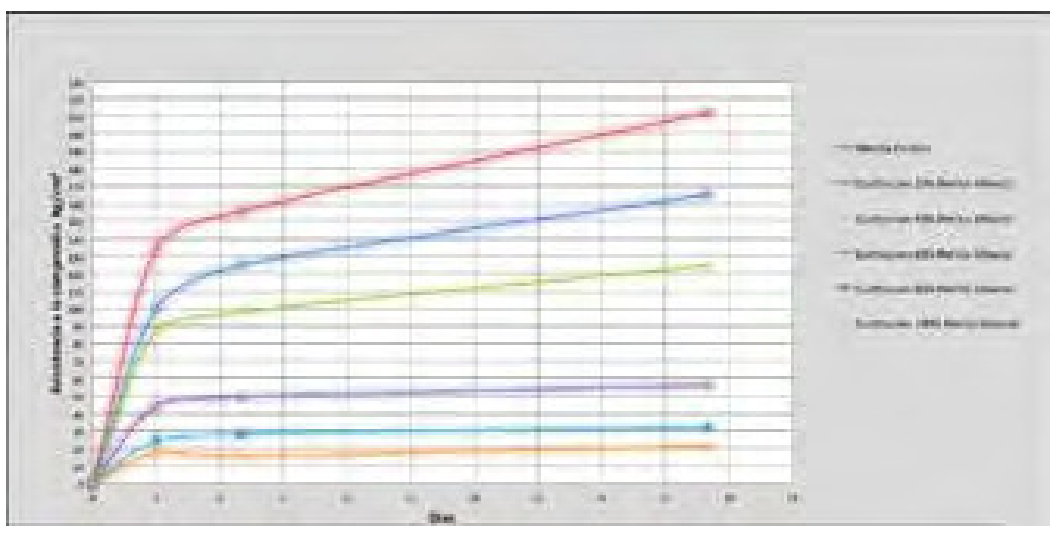

Figura 7. Relación resistencia a compresión y porcentaje de sustitución para perlita sintética.

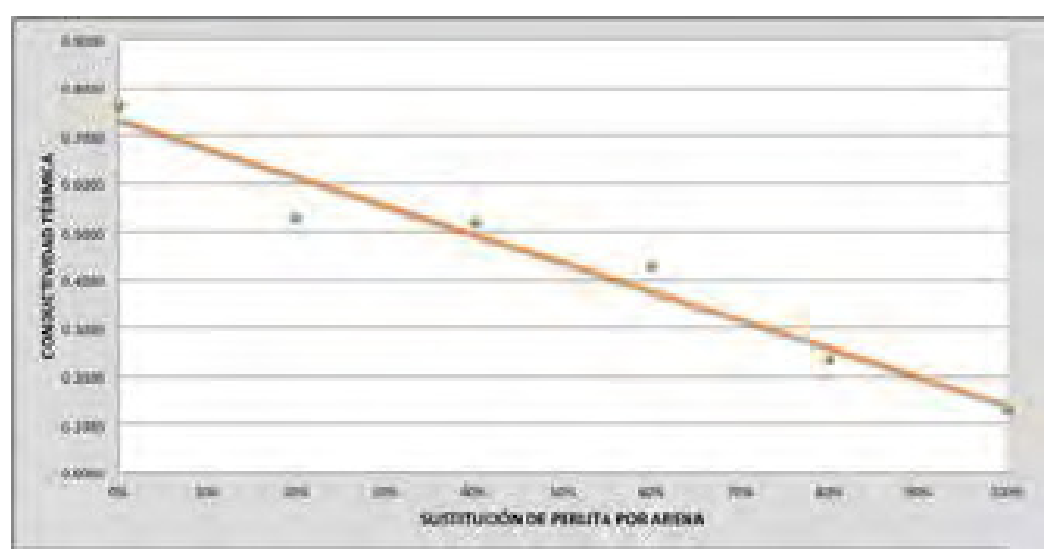

Figura 8. Relación entre conductividad térmica y porcentaje de sustitución 
En la figura 8, se observa que la conductividad térmica aumenta en la media que disminuye el porcentaje de sustitución.

De acuerdo la norma NMX-C-486-ONNCCE-2014, los morteros que se recomiendan para usos estructurales son aquellos que sobrepasan los valores de $75 \mathrm{~kg} / \mathrm{cm}^{2}$. De acuerdo a los resultados, los morteros que se pueden utilizar para estos efectos son los que corresponden al mortero base, además el de $20 \%, 40 \%$ de sustitución. Estos morteros aligerados ofrecen una reducción de hasta el $12 \%$ en la conductividad térmica, lo que significa un porcentaje equiparable en su capacidad de aislamiento, al ser utilizados en forma de emplastes o para la fabricación de tabiques con las debidas indicaciones técnicas.

\section{CONCLUSIONES}

De acuerdo a los resultados obtenidos de las pruebas realizadas a las mezclas en estado endurecido, se concluye que el aumento en la cantidad de materiales aligerantes disminuye la conductividad térmica, pero disminuye también la capacidad de soportar esfuerzo a la compresión. Esta situación nos lleva a concluir que al aligerar las mezclas debemos seleccionar aquellas que proporcionen una mejora en su capacidad aislante, pero sin sacrificar la capacidad de los materiales de soportar esfuerzos a la compresión, ya que de acuerdo a la normatividad mexicana las mezclas deben tener un mínimo de resistencia mecánica para poder determinar su utilización como materiales estructurales. Para este caso se concluye que las mezclas útiles para construcción de acuerdo la norma NMX-C-486-ONNCCE-2014, serían solo aquellos que cumplieran con un mínimo de $75 \mathrm{~kg} / \mathrm{cm}^{2}$ las cuales corresponden solo a las de sustitución de $0 \%, 20 \%$ y $40 \%$, el resto de las mezclas pueden ser utilizadas para rellenos y encofrados, los cuales elevan la capacidad de aislamiento hasta un $20 \%$ en relación al mortero base.

Estos resultados nos llevan a proponer que se continúe con los estudios del comportamiento térmico de estos morteros, pero ya inmersos en el estudio de una edificación, para evaluar los efectos que pueda tener su uso en cuanto a materiales que contribuyan a conservar el confort térmico interior o ahorros de energía en las edificaciones.

Agradecemos al Consejo Nacional de Ciencia y Tecnología y a la Comisión Nacional de Vivienda por el apoyo para la realización del proyecto denominado: "Diseño termofísico de componentes constructivos aligerados con características térmicas para elaborar muros de vivienda de interés social" CONAVI-2014-1236187.

\section{BIBLIOGRAFÍA}

[1] NMX-C-486-ONNCCE-2014. Norma Mexicana. Industria de la Construcción. Mortero para uso estructural.

[2] NMX-C-460-ENER-2009. Norma Mexicana. Industria de la
Construcción. Aislamiento térmico- Valor R para la envolvente de Vivienda por zona térmica para la republica mexicana. Especificaciones y verificación.

[3] Barros, A., Caballero, J. M., y Zaldo, V. (1981). Aplicaciones del Cemento Reforzado con Fibra de Vidrio. informes de la construcción, 33(333-34-335-336), 73-81.

[4] Bustamante, R., Mayor, P., Rangel, C., y Hernández, F. (2008). Propiedades térmicas, acústicas y mecánicas de placas de mortero caucho-cemento. Universidad Politécnica de Madrid, 1-9.

[5] Del Río, M., Santa Cruz, J., González, M. (2005). Morteros Aligerados con Arcilla Expandida: Influencia de la Granulometría y la Adición de Fibras de Vidrio AR en el Comportamiento Mecánico. informes de la construcción, 57(497), 39-46.

[6] Marco, J., García, E., Más, M.l., Alcaraz, V., y Luizaga, A. (2012). Estudio de la resistencia a compresión de morteros fabricados con conglomerante compuesto de polvo de vidrio. informes de la construcción, 64(528), 529-536.

[7] Durán Matas Cinthya Guadalupe. "Análisis termo- físico en mortero cemento arena 1:4 con sustitución en diferentes porcentajes de perlita mineral". Universidad de Sonora, Tesis de Licenciatura. Hermosillo Sonora México. Abril de 2017.

[8] D. Sánchez, Tecnología del concreto y mortero, México. Ed. BHANDER 2013.

[9] NMX-C-061-ONNCCE-2015. Norma Mexicana. Industria de la Construcción. Cementantes hidráulicos - Determinación de la resistencia a la compresión de cementantes hidráulicos.

[10] NMX-C-157-ONNCCE-2006. Norma Mexicana. Industria de la Construcción. Concreto-Determinación del contenido de aire del concreto fresco por el método de presión.

[11]. Design and Function of the Guarded Hot Plate Apparatus. Lambda Metter. Alemania.

[12] ASTM-C177-13. Norma Americana. Método de prueba estándar para mediciones de flujo de calor en estado estacionario y propiedades de transmisión térmica por medio del aparato de placa caliente protegida.

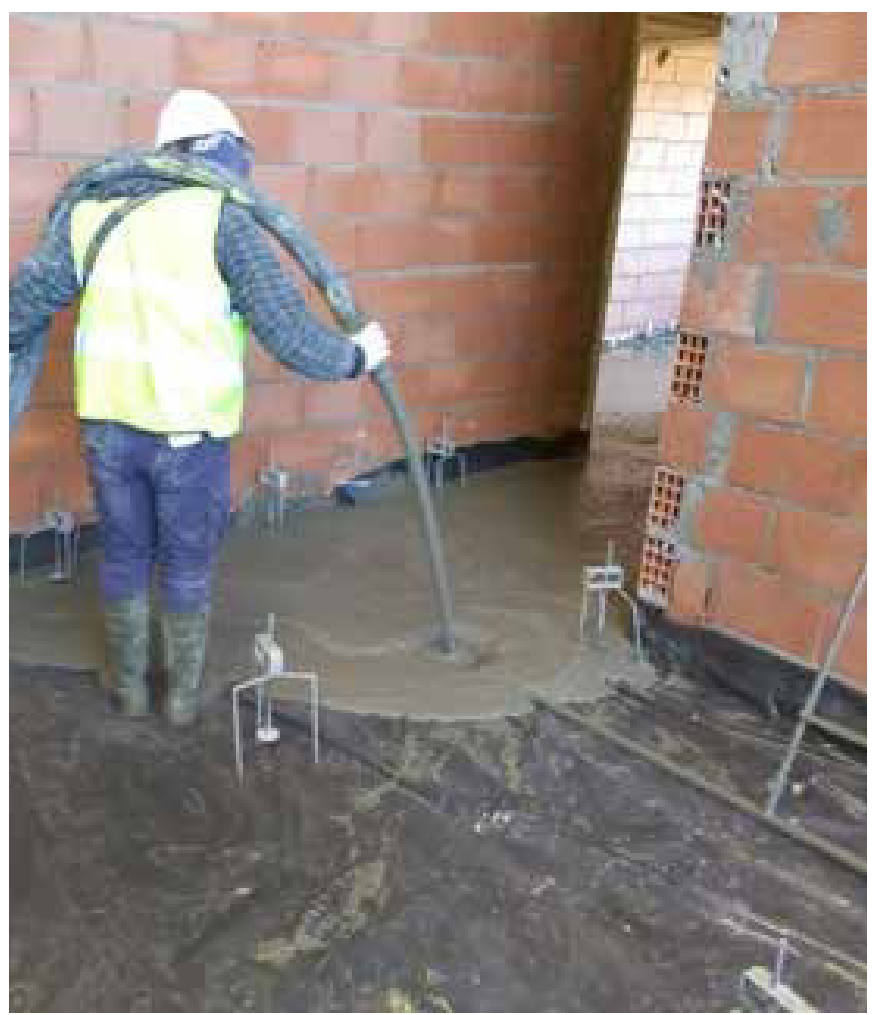

